Supporting Materials -1

\title{
New Polyamide Cryptand for Anion Binding
}

\author{
Sung Ok Kang, José M. Llinares, Douglas Powell, David VanderVelde \\ and Kristin Bowman-James* \\ Department of Chemistry, University of Kansas, Lawrence, Kansas 66045 \\ E-mail: kbowman-james@ku.edu
}

NMR studies:

${ }^{1} \mathrm{H}$ NMR spectra were recorded on a Bruker Avances 500 spectrometer at $500 \mathrm{MHz}$. Each titration was performed by 20 measurements in DMSO- $d_{6}$ at room temperature. Aliquots from stock solution of tetrabutylammonium salts $(20 \mathrm{mM})$ were gradually added to the initial solution of ligand $(2 \mathrm{mM})$. All proton signals were referred to TMS used in capillary tube. The association constants $K$ were calculated by non-linear regression curve fitting program with EQNMR. ${ }^{1}$ 


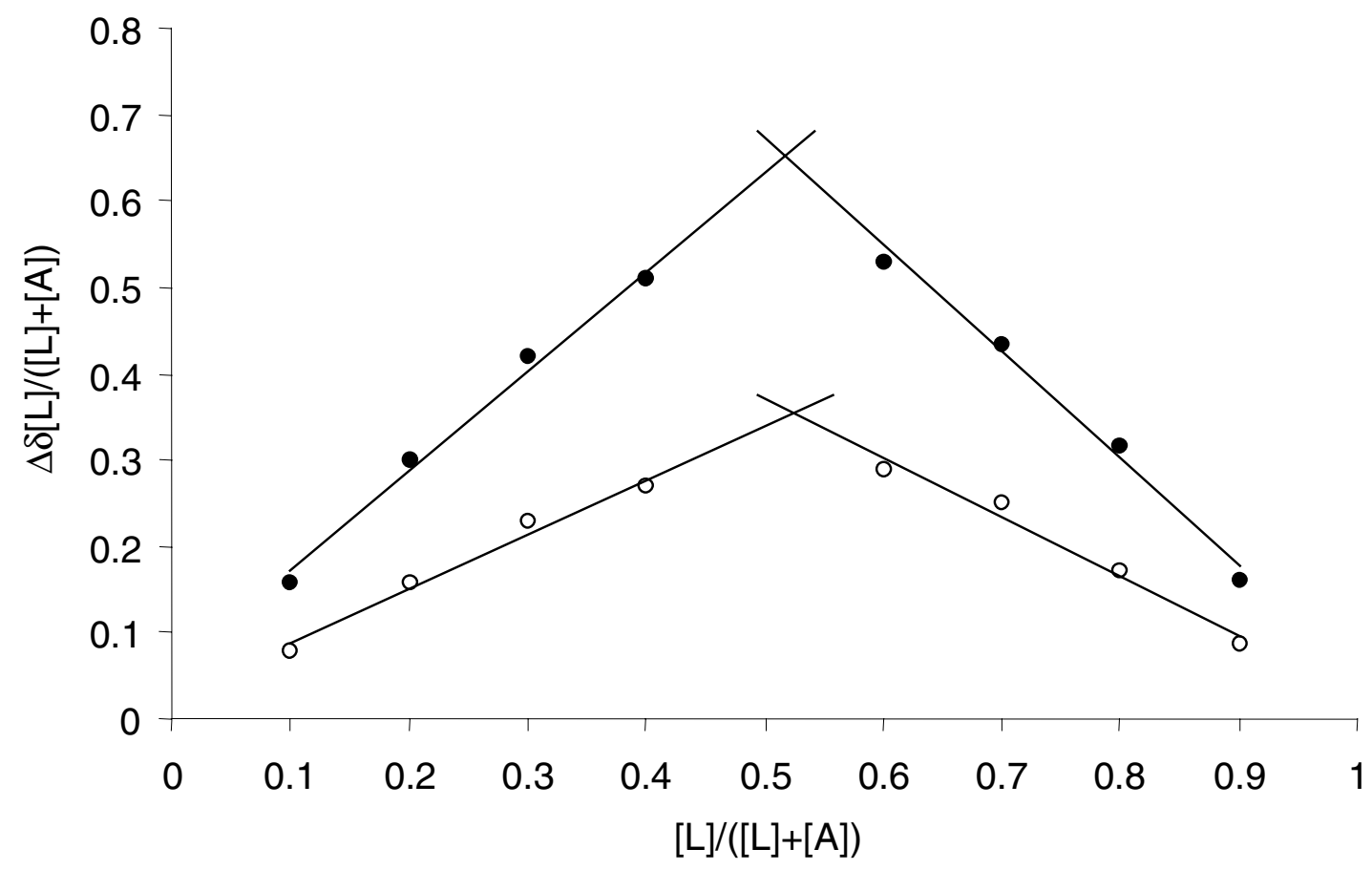

Figure 1. Job plots of $\mathbf{L}$ with $n-\mathrm{Bu}_{4} \mathrm{~N}^{+} \mathrm{Cl}{ }^{-}(\bullet)$ and $n-\mathrm{Bu}_{4} \mathrm{~N}^{+} \mathrm{H}_{2} \mathrm{PO}_{4}^{-}(\circ)$ in DMSO- $d_{6}$ at $25^{\circ} \mathrm{C}$. Total concentration $\left[\mathbf{L}+n-\mathrm{Bu}_{4} \mathrm{~N}^{+} \mathrm{A}^{-}\right]=2 \mathrm{mM}$. 


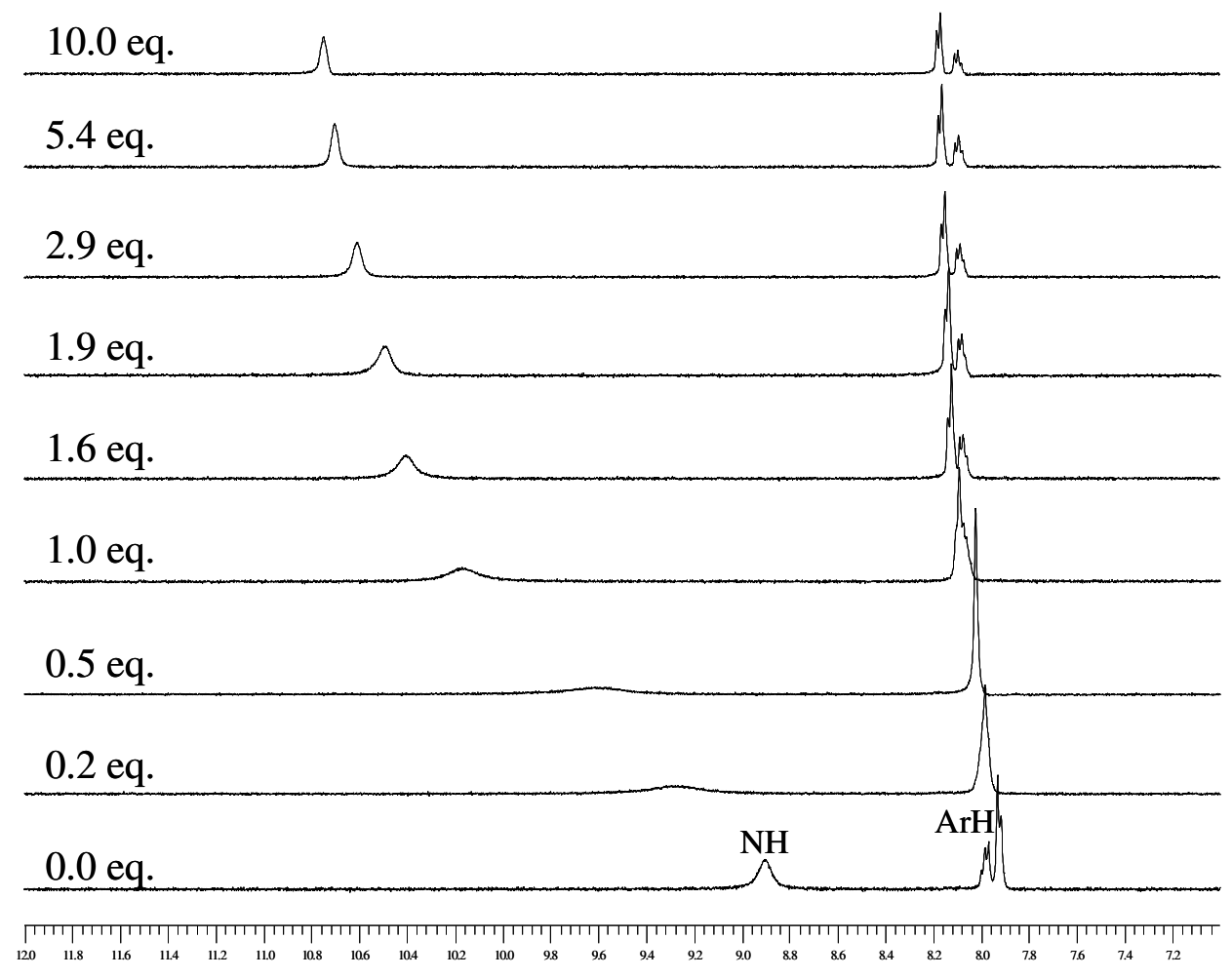

Figure 2. Partial ${ }^{1} \mathrm{H}$ NMR spectra of $\mathbf{L}$ in the presence of $n-\mathrm{Bu}_{4} \mathrm{~N}^{+} \mathrm{Cl}^{-}$in DMSO- $d_{6}$. Numbers at the left side indicate the equivalent amounts of $\mathrm{Cl}^{-}$added 


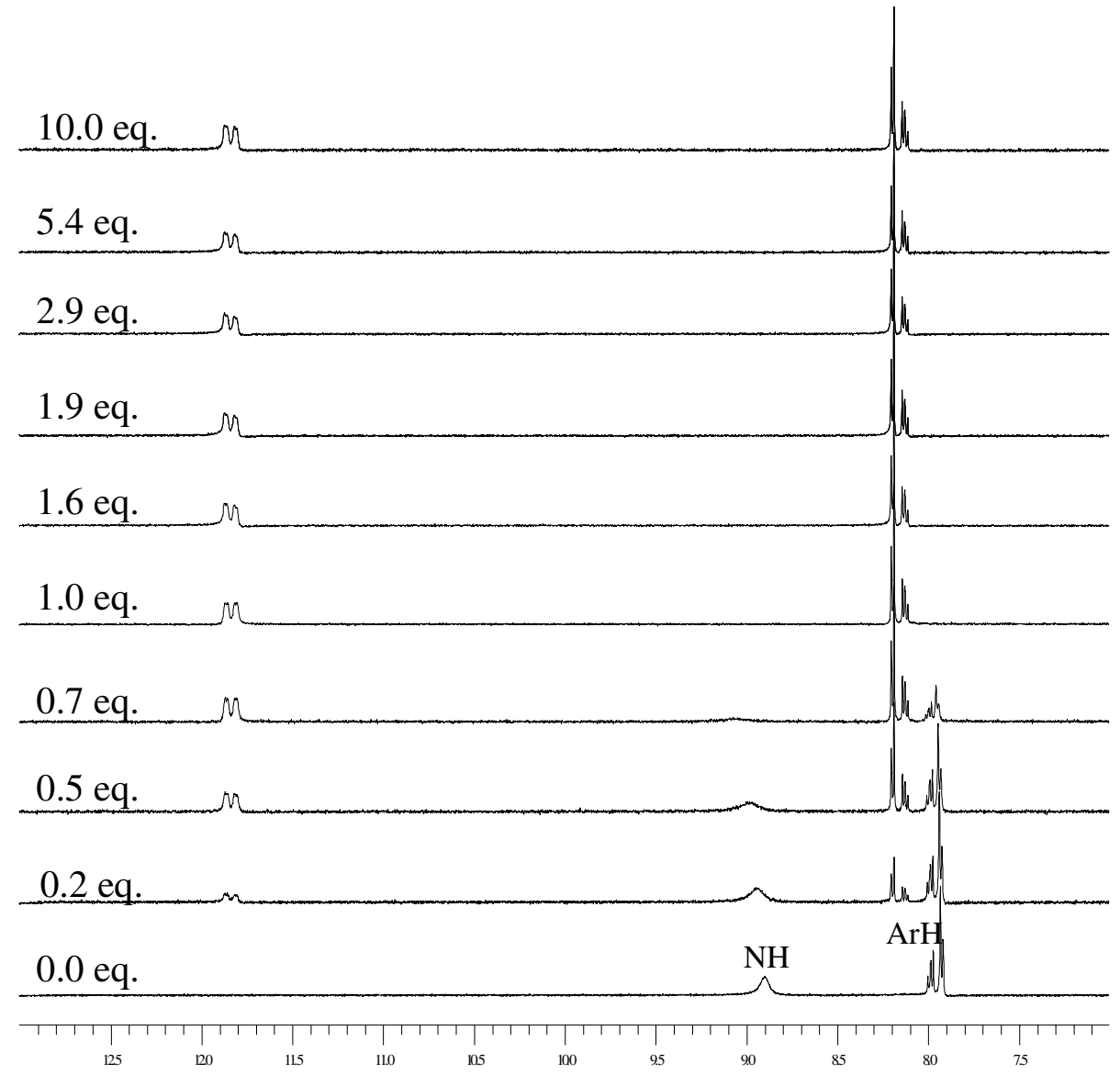

Figure 3. Partial ${ }^{1} \mathrm{H}$ NMR spectra of $\mathbf{L}$ in the presence of $n-\mathrm{Bu}_{4} \mathrm{~N}^{+} \mathrm{F}^{-}$in DMSO- $d_{6}$. Numbers at the left side indicate the equivalent amounts of $\mathrm{F}^{-}$added. 